\title{
Linkage of monogenic infantile hypertrophic pyloric stenosis to chromosome $16 \mathrm{q} 24$
}

\author{
Kate V Everett*,1 ${ }^{*}$ Francesca Capon ${ }^{1,4}$, Christina Georgoula ${ }^{1}$, Barry A Chioza ${ }^{1}$, Ashley Reece ${ }^{1}$, \\ Mervyn Jaswon ${ }^{2}$, Agostino Pierro ${ }^{1}$, Prem Puri ${ }^{3}$, R Mark Gardiner ${ }^{1}$ and Eddie MK Chung ${ }^{1}$ \\ ${ }^{1}$ University College London Institute of Child Health, London, UK; ${ }^{2}$ The Whittington Hospital NHS Trust, London, UK; \\ ${ }^{3}$ Our Lady's Hospital for Sick Children, Dublin, Ireland
}

Infantile hypertrophic pyloric stenosis (IHPS) is the most common inherited form of gastrointestinal obstruction in infancy. The disease is considered a paradigm for the sex-modified model of multifactorial inheritance and affects males four times more frequently than females. However, extended pedigrees consistent with autosomal dominant inheritance have been documented. We have analysed data from an extended IHPS family including eight affected individuals (five males and three females) and mapped the disease locus to chromosome 16q24 (LOD score $=3.7$ ) through an SNP-based genome wide scan. Fourteen additional multiplex pedigrees did not show evidence of linkage to this region, indicating locus heterogeneity.

European Journal of Human Genetics (2008) 16, 1151-1154; doi:10.1038/ejhg.2008.86; published online 14 May 2008

Keywords: pyloric stenosis; linkage; chromosome 16q24

\section{Introduction}

Infantile hypertrophic pyloric stenosis (IHPS, No. 179010) is the most common inherited cause of gastrointestinal obstruction in the first few months of life. The incidence of IHPS is $1-8$ per 1000 live births in the Caucasian population. It presents in infants 2-6 weeks after birth with projectile vomiting, weight loss and dehydration. Standard treatment is by pyloromyotomy (for review, see MacMahon $^{1}$ ).

IHPS shows familial aggregation and is a paradigm for the multifactorial sex-modified threshold model of inheritance, with affected males outnumbering females in a $4: 1$ ratio. ${ }^{2}$ IHPS is predicted to be oligogenic, determined by two or three loci of moderate effect conferring individual genotype relative risks up to $5 .^{3}$ Genetic and functional

*Correspondence: Dr KV Everett, General and Adolescent Paediatric Unit, Centre for Human Molecular Genetics, UCL Institute of Child Health, the Rayne Building, 5 University Street, London WC1E 6JJ, UK.

Tel: + 440207 6796124; Fax: + 440207 6796103;

E-mail: kate.everett@ucl.ac.uk

${ }^{4}$ Present affiliation: King's College School of Medicine, London, UK Received 10 January 2008; revised 27 March 2008; accepted 28 March 2008; published online 14 May 2008 analyses indicate that the NOS1 gene (MIM No. 163731) may be a predisposing locus for IHPS (IHPS1, MIM No. 179010). ${ }^{4,5}$ A single nucleotide polymorphism (SNP)-based genome-wide scan in 81 families (each with at least two individuals with IHPS) has demonstrated linkage to chromosomes 11 and $\mathrm{X}^{6}$

IHPS has been associated with several genetic syndromes $^{7,8}$ and chromosomal abnormalities. ${ }^{9,10}$ Autosomal dominant monogenic forms of IHPS have also been reported in several extended pedigrees. ${ }^{11-13}$ We have previously mapped the first locus for monogenic IHPS (IHPS2, MIM \#610260) to chromosome 16p12-p13 in a multigeneration pedigree with 10 affected individuals. Locus heterogeneity exists as 10 additional extended pedigrees did not show evidence of linkage to the 16p13-p12 locus. ${ }^{13}$

We report here the identification of a second monogenic locus for IHPS in a large multigeneration pedigree in which IHPS is inherited as an autosomal dominant trait. Linkage has been excluded to both 16p12-p13 and NOS1 in this family. ${ }^{13}$ We provide further evidence for locus heterogeneity in IHPS. 


\section{Materials and methods Subjects and samples}

Fifteen Caucasian multiplex pedigrees were ascertained from paediatric units in the United Kingdom and Ireland. Family IHPS078 (Figure 1) is an extended pedigree, which has been described previously; ${ }^{13}$ DNA is available for a total of 16 individuals from this family, including seven affected individuals. The other 14 pedigrees each had at least four affected individuals (Supplementary Figure 1S). The study was approved by the Ethics Committee of University College London Hospital and by the appropriate institutional review boards of all other participating hospitals. Informed consent was obtained from all participating individuals.

\section{Linkage analysis}

We undertook a genome-wide scan in IHPS078 using the Illumina Linkage Panel IV. Samples were genotyped using the panel of 5850 SNPs spaced at an average distance of $0.64 \mathrm{cM}$ (Illumina Inc., San Diego CA, USA, BeadArray platform and GoldenGate assay). The maximum LOD score expected for this family under simulation using FastSLINK ${ }^{14,15}$ was 3.7 (data not shown).

Parametric linkage analyses were performed using MERLIN 1.0.1 ${ }^{16}$ assuming autosomal dominant inheritance with $65 \%$ penetrance. The disease allele frequency was set at 0.001 and phenocopy rate at 0.0001 . These values are conservative estimates designed to be compatible with the observed population prevalence (disease allele frequency) as well as the transmission pattern in family IHPS078 (reflected by the reduced penetrance). Marker allele frequencies were taken from the Caucasian control samples provided by Illumina. Haplotypes were determined using the 'best' option in MERLIN 1.0.1, which estimates haplotypes based on the most likely pattern of gene flow.

Four microsatellite markers (D16S3048, D16S2625, D16S520 and D16S3026; primer sequences and cycling conditions available on request) were typed in the other 14 Caucasian multiplex pedigrees using an ABI PRISM ${ }^{\circledR} 373$ DNA sequencer (Applied Biosystems).

\section{Candidate gene analysis}

We investigated the function and expression patterns of all transcripts in linked regions by mining the Ensembl, UniGene and GeneCards databases. We examined all coding and UTR exons, as well as exon-intron junctions (sequencing $100 \mathrm{bp}$ either side of each exon), in the best functional candidate, using the affected individual IV.1. Purified PCR products (primer sequences and cycling conditions available on request) were sequenced using an ABI PRISM 373 DNA sequencer (Applied Biosystems).

\section{Results \\ Linkage analysis \\ Multipoint LOD scores $\geq 3$ were observed at a single genomic region on chromosome 16q24 upon genome- wide linkage analysis of family IHPS078 (maximum $\mathrm{LOD}=3.7$; Figure 2; see Supplementary Table $1 \mathrm{~S}$ for summary of results for all autosomes). The critical region interval identified by the genome-wide scan and defined by observed recombination events spans $4.2 \mathrm{Mb}$. All affected individuals shared a common haplotype across this disease interval (Figure 2; Supplementary Figure 2S). There are multiple LD blocks across this interval (as defined by Barrett et al; ${ }^{17}$ data not shown). \\ The chromosome 16q microsatellite markers, which spanned the critical region (D16S3048, D16S2625, D16S520 and D16S3026) yielded negative LOD scores in the additional 14 families examined with the exception of a single pedigree, which yielded positive but non-signi- ficant LOD scores (family IHPS004, maximum $\mathrm{LOD}=0.5$; see Supplementary Table 2S).}

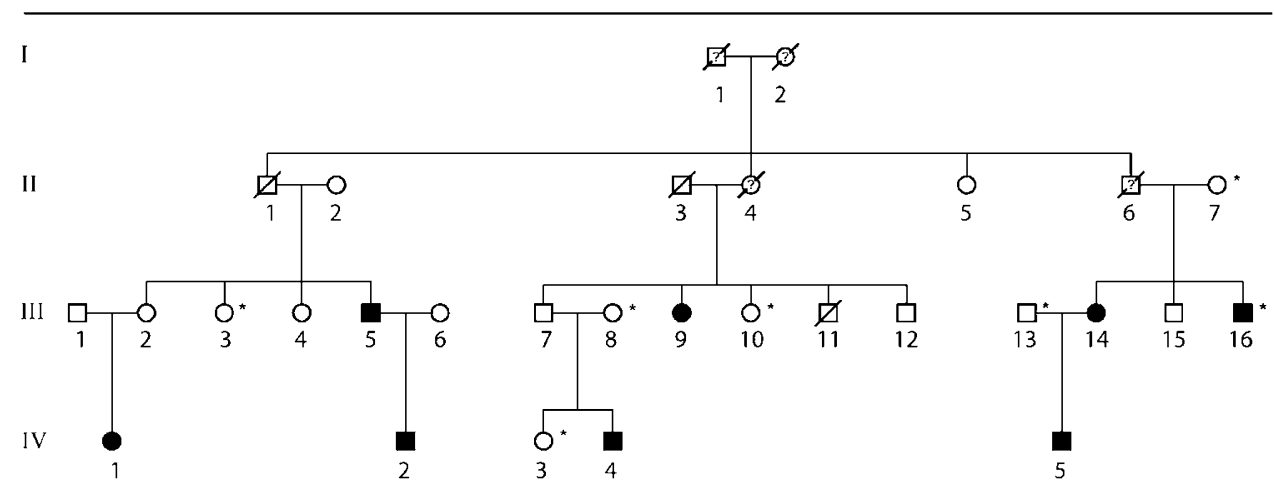

Figure 1 Structure of family IHPS078. Biological samples were obtained from and genotyping performed on all living individuals, except those marked with an asterisk (*). No samples were obtained from any of the deceased family members. Question marks (?) indicate individuals whose disease status could not be determined. Affected individuals are shaded. 


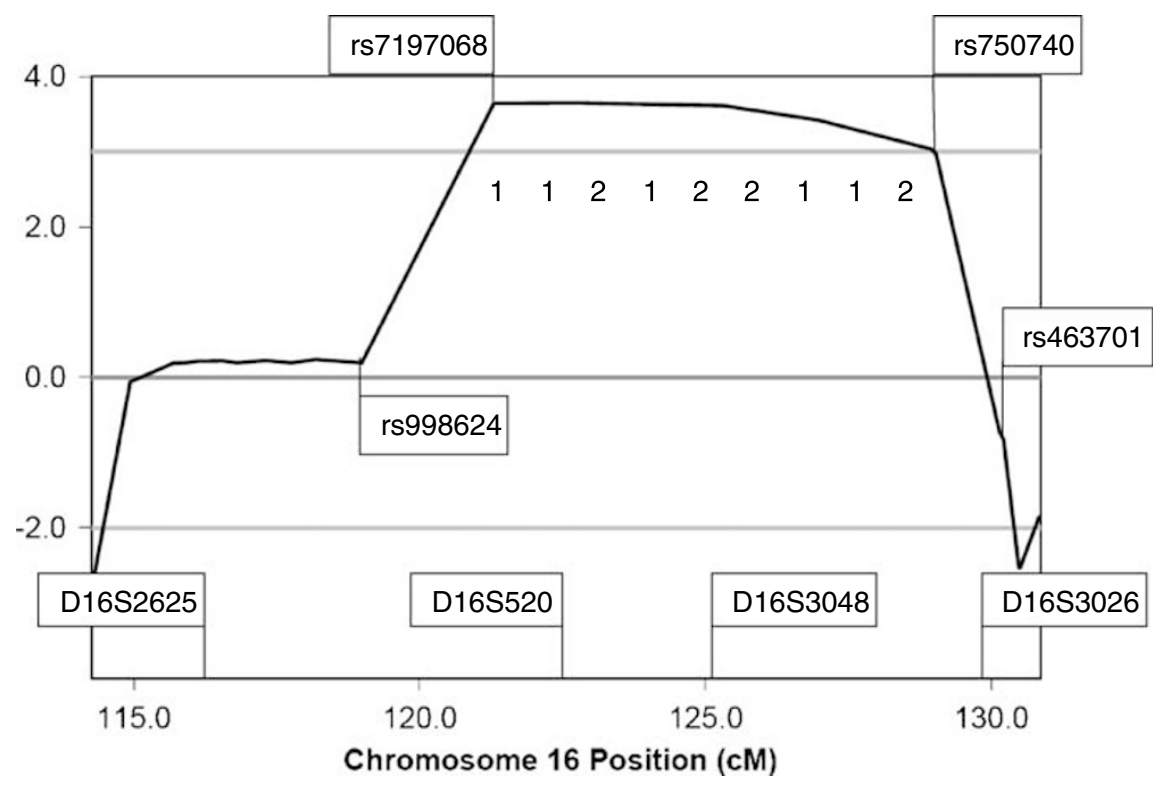

Figure 2 Output of chromosome 16 multipoint LOD score analysis. The position of those SNPs flanking the points of recombination are shown. The haplotype shared by all affected individuals across the disease interval is also given. The genetic positions of the four microsatellite markers used are also shown.

\section{Candidate gene analysis}

The critical region contained a total of 68 known or predicted genes. We prioritised for mutational analysis the SLC7A5 (MIM 600182) gene. SLC7A5 encodes a member of the L amino-acid transporter family, LAT1, which has a role in the regulation of smooth muscle contraction. ${ }^{18}$ Resequencing of all coding and UTR exons, as well as exonintron junctions, in $S L C 7 A 5$, using the affected individual IV.1 did not identify and pathogenic mutations.

\section{Discussion}

We have mapped a second locus for monogenic IHPS through an SNP-based genome scan of a multigenerational pedigree. The sex-segregation male-to-female ratio in family IHPS078 is 1.7:1. In our resource, the IHPS male sex bias tends to be less pronounced in familial cases (2.2:1 in the 14 additional families used in this study) than in sporadic cases (5.2:1 in our resource of 358 trios; data not shown). This suggests that underlying sex-specific factors are of greater importance in sporadic cases, or that familial cases occur because of a more highly penetrant allele to which females do not have a sex-specific protection. The analysis of an additional 14 families yielded negative or non-significant LOD scores, indicating that the chromosome $16 \mathrm{q}$ locus is not likely to contribute to a high proportion of IHPS cases. Although consistent with linkage in one family, this family alone does not have the power to attain statistical significance (expected maximum LOD for family IHPSO04 is 1.3 using 1000 simulations in Allegro1.2c; ${ }^{19}$ data not shown). Because IHPS004 did not produce a linkage result close to its expected maximum LOD score, we decided to focus initial resequencing efforts on family IHPS078 alone.

Genetic heterogeneity in a Mendelian subtype of a complex disease is common. In the case of IHPS, the presence of genetic heterogeneity is consistent with the complexity of the molecular pathways underlying smooth muscle relaxation. ${ }^{20}$ Given the high number of proteins involved in these signalling cascades, it is reasonable to hypothesise that IHPS may be caused by mutations occurring in different genes and segregating with variable degrees of penetrance. The results of this study, combined with those of Capon et al, ${ }^{13}$ provide further support for this hypothesis. Two large multigenerational pedigrees have now provided evidence for the existence of two susceptibility loci in monogenic IHPS, probably specific to those particular families. The critical region interval identified by the genome-wide scan of family IHPS078 spans $4.2 \mathrm{Mb}$ on 16q24. We prioritised the SLC7A5 (MIM 600182) gene for mutational analysis. SLC7A5 encodes LAT1. It occurs as a heterodimer and is proposed to transport extracellular S-nitrosocysteine (CSNO) into the cell where it transduces nitric oxide activity. ${ }^{18,21,22}$ In this way, LAT1 has a role to play in the regulation of smooth muscle contraction. Overexpression of LAT1 in mammalian cells has been shown to result in an increased uptake of L-CSNO, whereas knockdown of LAT1 leads to a decreased uptake. ${ }^{18}$ Sequencing failed to identify pathogenic mutations, but this does not exclude the occurrence of regulatory variants in the promoter or deep intronic regions. It is possible that other positional candidate genes will need to be sequenced 
to identify the pathogenic mutation. The linkage interval identified in family IHPS078 contains at least 30 transcripts of unknown function from which further IHPS candidates may emerge as further information becomes available.

Although our findings indicate that chromosome 16q24 is not a common monogenic IHPS locus, the eventual identification of the disease gene will allow an assessment of the contribution of chromosome 16q24 mutations to sporadic IHPS as well as suggesting potential relevant biological pathways to assess further functional candidates.

\section{Acknowledgements}

We thank all the families who participated in this study. We are grateful to $K$ Parker for technical assistance and to K Rogers and $J$ Mulligan for their contribution to ascertainment work. This work was funded by Birth Defects Foundation Newlife and Action Medical Research.

\section{Web Resources}

The URLs for data presented herein are as follows:

Ensembl, http://www.ensembl.org/Homo_sapiens/index.html GeneCards, http://www.genecards.org/index.shtml

Online Mendelian Inheritance in Man (OMIM), http:// www.ncbi.nlm.nih.gov/omim/

UniGene, http://www.ncbi.nlm.nih.gov/sites/entrez?db=unigene

\section{References}

1 MacMahon B: The continuing enigma of pyloric stenosis of infancy: a review. Epidemiology 2006; 17: 195-201.

2 Carter CO: The inheritance of congenital pyloric stenosis. $\mathrm{Br}$ Med Bull 1961; 17: 251-254.

3 Mitchell LE, Risch N: The genetics of infantile hypertrophic pyloric stenosis. A reanalysis. Am J Dis Child 1993; 147: $1203-1211$.

4 Chung E, Curtis D, Chen G et al: Genetic evidence for the neuronal nitric oxide synthase gene (NOS1) as a susceptibility locus for infantile pyloric stenosis. Am J Hum Genet 1996; 58: $363-370$.

5 Saur D, Vanderwinden JM, Seidler B, Schmid RM, De Laet MH, Allescher HD: Single-nucleotide promoter polymorphism alters transcription of neuronal nitric oxide synthase exon 1c in infantile hypertrophic pyloric stenosis. Proc Natl Acad Sci USA 2004; 101: 1662-1667.

6 Everett KV, Chioza BA, Georgoula C et al: Genome-wide highdensity SNP-based linkage analysis of infantile hypertrophic pyloric stenosis identifies loci on chromosomes 11q14-q22 and Xq23. Am J Hum Genet 2008; 82: 756-762.

7 Jackson L, Kline AD, Barr MA, Koch S: de Lange syndrome: a clinical review of 310 individuals. Am J Med Genet 1993; 47: 940-946.

8 Smith DW, Lemli L, Opitz JM: A newly recognised syndrome of multiple congenital anomalies. J Pediatr 1964; 64: 210-217.

9 Heller A, Seidel J, Hubler A et al: Molecular cytogenetic characterisation of partial trisomy $9 \mathrm{q}$ in a case with pyloric stenosis and a review. J Med Genet 2000; 37: 529-532.

10 Hodgson SV, Berry AC, Dunbar HM: Two brothers with an unbalanced 8;17 translocation and infantile pyloric stenosis. Clin Genet 1995; 48: 328-330.

11 Finsen VR: Infantile hypertrophic pyloric stenosis - unusual familial incidence. Arch Dis Child 1979; 54: 720-721.

12 Fried K, Aviv S, Nisenbaum C: Probable autosomal dominant infantile pyloric stenosis in a large kindred. Clin Genet 1981; 20: 328-330.

13 Capon F, Reece A, Ravindrarajah R, Chung E: Linkage of monogenic infantile hypertrophic pyloric stenosis to chromosome 16p12-p13 and evidence for genetic heterogeneity. Am J Hum Genet 2006; 79: 378-382.

14 Ott J: Computer-simulation methods in human linkage analysis. Proc Natl Acad Sci USA 1989; 86: 4175-4178.

15 Weeks DE, Ott J, Lathrop GM: SLINK: a general simulation program for linkage analysis. Am J Hum Genet 1990; 47: A204 (abstr).

16 Abecasis GR, Cherny SS, Cookson WO, Cardon LR: Merlin - rapid analysis of dense genetic maps using sparse gene flow trees. Nat Genet 2002; 30: 97-101.

17 Barrett JC, Fry B, Maller J, Daly MJ: Haploview: analysis and visualization of $\mathrm{LD}$ and haplotype maps. Bioinformatics 2005; 21: $263-265$.

18 Li S, Whorton AR: Identification of stereoselective transporters for S-nitroso-L-cysteine: role of LAT1 and LAT2 in biological activity of S-nitrosothiols. J Biol Chem 2005; 280: 20102-20110.

19 Gudbjartsson DF, Jonasson K, Frigge ML, Kong A: Allegro a new computer program for multipoint linkage analysis. Nat Genet 2000; 25: 12-13.

20 Schlossmann J, Feil R, Hofmann F: Signaling through NO and cGMP-dependent protein kinases. Ann Med 2003; 35: 21-27.

21 Foster MW, McMahon TJ, Stamler JS: S-nitrosylation in health and disease. Trends Mol Med 2003; 9: 160-168.

22 Li S, Whorton AR: Functional characterization of two S-nitroso-Lcysteine transporters, which mediate movement of NO equivalents into vascular cells. Am J Physiol Cell Physiol 2007; 292: C1263-C1271.

Supplementary Information accompanies the paper on European Journal of Human Genetics website (http://www.nature.com/ejhg) 